\title{
Transient silencing mediated by in vitro synthesized double-stranded RNA indicates that PsCdc14 is required for sporangial development in a soybean root rot pathogen
}

\author{
ZHAO Wei, YANG XinYu, DONG SuoMeng, SHENG YuTing, WANG YuanChao \& \\ ZHENG XiaoBo*
}

College of Plant Protection, Key Laboratory of Integrated Management of Crop Diseases and Pests, Ministry of Education of China, Nanjing Agricultural University, Nanjing 210095, China

Received June 19, 2011; accepted October 18, 2011

\begin{abstract}
In many eukaryotic organisms, Cdc14 phosphatase regulates multiple biological events during anaphase and is essential for mitosis. It has been shown that $\mathrm{Cdc} 14$ is required for sporulation in the potato blight pathogen Phytophthora infestans; however, the role that the $\mathrm{Cdc} 14$ homolog (PsCdc14) plays in the soil-borne soybean root rot pathogen $P$. sojae remains ambiguous. $P s C d c 14$ is highly expressed in sporulation, zoospore, and cyst life stages, but not in vegetative mycelia and infection stages, suggesting that it contributes to asexual reproduction and thus the spread of the disease. Double-stranded RNA (dsRNA) mediates gene silencing, a post-transcriptional and highly conserved process in eukaryotes, involving specific gene silencing through degradation of target mRNA. We combined in vitro dsRNA synthesis and a polyethylene glycol-mediated transformation system to construct a dsRNA-mediated transient gene silencing system; and then performed a functional analysis of PsCdc14 in $P$. sojae. PsCdc14 mRNA was dramatically reduced in transformants after protoplasts were exposed in in vitro synthesized $P s C d c 14$ dsRNA, resulting in low sporangial production and abnormal development in $P$. sojae silencing lines. Furthermore, dsRNA-mediated transient gene silencing could enable elucidation of $P$. sojae rapid gene function, facilitating our understanding of the development and pathogenicity mechanisms of this oomycete fungus.
\end{abstract}

Phytophthora sojae, PsCdc14, sporangium, transient gene silencing

Citation: Zhao W, Yang X Y, Dong S M, et al. Transient silencing mediated by in vitro synthesized double-stranded RNA indicates that PsCde14 is required for sporangial development in a soybean root rot pathogen. Sci China Life Sci, 2011, 54: 1143-1150, doi: 10.1007/s11427-011-4250-2

Phytophthora sojae, one of more than 80 species of Phytophthora that causes notorious diseases on a wide range of agriculturally and ornamentally important plants, is the casual agent of stem and root rot in soybean. P. sojae is responsible for annual yield losses estimated to be USD 1-2 billion worldwide [1,2]. This oomycete fungus is a hemibiotroph and is homothallic, propagating through clonal zoospores and sexual oospores, producing nondehiscent sporangia when soils are saturated [3]. The development and spread of zoospores and subsequent infection of soybean

*Corresponding author (email: xbzheng@ njau.edu.cn) roots is favored by saturated soil conditions [4]. Oospores are readily produced in susceptible soybean roots and can survive in crop residues and soil for many years [5].

An important feature of $P$. sojae is the formation of the sporangia during asexual development, the central part of the disease cycle of this pathogen. Sporangia develop on the termini of specialized aerial hyphae if humidity and oxygen levels are high. Although in some species such as $P$. infestans, sporangia are released freely from aerial hyphae and serve as agents of dispersal, the sporangia of $P$. sojae are not readily released from the hyphae. The asexual sporangia of $P$. sojae have a remarkable ability to germinate in 
two different ways. At higher temperatures, direct germination occurs, and hyphae emerge through the sporangial wall. Plant colonization can then occur through openings in a host such as stomata, lenticels, or wounds. Indirect germination, also known as zoosporogenesis, is predominant at cooler temperatures [3,6]. Relatively little is known about the molecular biology of zoospore formation or germination, although a few participant genes have been studied in Phytophthora $[7,8]$.

Coordinating nuclear division with growth and the cell cycle is central to the development of eukaryotes. The transmission of genetic information from one generation to the next requires the accurate replication of DNA during S-phase, and the faithful partitioning of chromosomes during mitosis requires the coordination of several cellular events. $C D C 14$ is one of the key regulators. CDC14 encodes a protein phosphatase that is essential for mitotic exit and meiotic progression [9-11]. Cdc14p dephosphorylates key mitotic targets, leading to the coordinated inactivation of mitotic cyclins, proper spindle disassembly, and completion of cytokinesis [12-14]. During the meiotic cell cycle, Cdc14p has been proposed to coordinate spindle disassembly and the two consecutive chromosome segregation events $[15,16]$. During most of the mitotic cell cycle, Cdc14p is kept inactive and sequestered in the nucleolus. Released from the nucleolus, Cdc14p can dephosphorylate $\mathrm{S}$-phase and M-phase mitotic cyclin substrates to coordinate the metaphase to anaphase transition [17-19]. The return of Cdc14p to the nucleolus signals the completion of mitosis [20,21]. Multiple regulatory pathways have been proposed to regulate $\mathrm{Cdc} 14 \mathrm{p}$ phosphatase activity during meiosis as well [22,23]. Cdc14p belongs to a subfamily of dual-specificity protein phosphatases that can dephosphorylate phosphotyrosine and phosphoserine/phosphothreonine residues [24]. Cdc14p is conserved in fungi, worms, and mammals [25,26]. In P. infestans, piCdc14 complemented a mutation in its $S$. cerevisiae ortholog, suggesting that it could be a mitotic regulator; however, the gene exhibits a pattern of expression strikingly different from that observed in yeast and other studied species. piCdcl4 is not transcribed during normal hyphal growth but is expressed only during the formation of sporangia, and appears required for sporulation. These results can be placed in the context of the adaptation of Cdc14 functions to different modes of growth during eukaryotic evolution [7].

Exposure of eukaryotic cells to double-stranded RNA (dsRNA) led to post-transcriptional degradation of homologous mRNA sequences [27]. Known as RNA interference (RNAi), this process was initially described in nematodes and subsequently demonstrated in fungi, plants, insects, and animals [27,28]. The dsRNA molecules that trigger RNAi can be delivered into cells by microinjection, transfection, or transformation. In P. infestans, transient silencing mediated by in vitro-synthesized dsRNA has been reported
[29-32]. Target genes were silenced from 12 to 17 days after the $P$. infestans protoplasts were exposed to dsRNA, and the gene silencing triggered by dsRNA was sequencespecific [32]. These reports show that dsRNA-mediated transient gene silencing can be used to generate detectable phenotypes and should provide a valuable tool for functional genomics in Phytophthora.

Our analysis of the transcription of $\mathrm{PsCdcl} 4$ in $\mathrm{P}$. sojae indicated that it is highly upregulated during sporulation, zoospore, and cyst stages. We combined in vitro dsRNA synthesis and a polyethylene glycol (PEG)-mediated transformation system to construct a dsRNA-mediated transient gene silencing system, and then performed a functional analysis of PsCdc14 in P. sojae.

\section{Materials and methods}

\subsection{Growth of Phytophthora sojae life cycle stages and plant inoculation}

$P$. sojae strain $\mathrm{P} 6497$ and all transgenic $P$. sojae isolates used were routinely grown on $10 \% \mathrm{~V} 8$ media at $25^{\circ} \mathrm{C}$ in the dark, as described by Erwin [2]. To obtain axenically prepared mycelium, hyphal tip plugs of P6497 were used to inoculate $30 \mathrm{~mL}$ of sterile clarified $10 \% \mathrm{~V} 8$ broth in $90 \mathrm{~mm}$ petri dishes. Stationary mycelial cultures were incubated at $25^{\circ} \mathrm{C}$ in the dark for $3 \mathrm{~d}$. Sporulating hyphae were prepared by repeatedly washing 2-day-old hyphae incubated in $10 \%$ V8 broth with sterile distilled water, and incubating the washed hyphae in the dark at $25^{\circ} \mathrm{C}$ for $4-8 \mathrm{~h}$ until sporangia had developed on most hyphae. Zoospores were filtered using Miracloth (Calbiochem) and collected by centrifugation at $2000 \times g$ for $2 \mathrm{~min}$. Cysts were obtained by vortexing a zoospore suspension for $30 \mathrm{~s}$ and then centrifuging at $2000 \times g$ for $2 \mathrm{~min}$. Cysts were germinated in clarified $5 \%$ V8 broth for $2 \mathrm{~h}$. The inoculation of soybean leaves with $P$. sojae mycelia, used for qRT-PCR analysis, was performed as described by Chen [33]. Mycelia were harvested from infected leaves at 1.5 hours post infection (hpi), and at $3 \mathrm{hpi}$, and then infected leaves were harvested at 6, 12, 24, and 48 hpi. All collected samples were immediately frozen in liquid nitrogen and stored at $-70^{\circ} \mathrm{C}$ prior to RNA extraction.

\subsection{RNA extraction}

Total RNA from various stages of the $P$. sojae life cycle was extracted from frozen samples ground in liquid nitrogen using a NucleoSpin RNA II RNA extraction kit (MachereyNagel), following the manufacturer's protocol. The integrity of the RNA was tested by agarose gel electrophoresis. Prior to cDNA synthesis, all RNA samples were treated with DNase I (Takara, D2270), following the manufacturer's protocol. First strand cDNA was synthesized from 1 to $5 \mu \mathrm{g}$ of total RNA by oligo (dT) priming using a M-MLV reverse transcriptase kit (Invitrogen), following the manufacturer's 
protocol.

\section{3 qRT-PCR analysis of gene expression}

For gene expression analysis, SYBR green qRT-PCR assays were performed. Primer pairs (Table 1) were designed to anneal specifically to each of the genes for qRT-PCR analysis. The actA gene from $P$. sojae was used as a constitutively expressed endogenous control, and the expression of each gene was determined relative to actA using the $\Delta \Delta C_{\mathrm{t}}$ method [30]. Expression of $P s C d c 14$ at different life cycle stages was compared with the level of its expression in a calibrated sample of cDNA from a mycelium. Although there was a high expression of $\mathrm{PsCdc14}$ in sporulating mycelia, zoospores, and cysts, the basal levels of $P s C d c 14$ mRNA in mycelia could be detected using the sensitivity of real time PCR. The expression of PsCdcl4 in the mycelial cDNA sample was assigned the value of 1.0. For expression of each gene in potentially silenced lines, the calibrated sample was the expression value from the wild type strain P6497 (WT) for each gene; this expression number was assigned the value of 1.0. qRT-PCR assays for gene expression were carried out using three biological replicates.

\subsection{Transformation of $P$. sojae}

We transformed $P$. sojae using a polyethylene glycol (PEG)-mediated protoplast transformation strategy [34] with the following modifications: Two-day-old $P$. sojae mycelial mats, cultured in pea broth medium, were rinsed and washed in $0.8 \mathrm{~mol} \mathrm{~L}^{-1}$ mannitol, then placed in enzyme solution $\left(0.4 \mathrm{~mol} \mathrm{~L}^{-1}\right.$ mannitol, $20 \mathrm{mmol} \mathrm{L}^{-1} \mathrm{KCl}, 20 \mathrm{mmol}$ $\mathrm{L}^{-1}$ MES (2-(N-Morpholino)ethanesulfonic acid hydrate), pH 5.7, $10 \mathrm{mmol} \mathrm{L}^{-1} \mathrm{CaCl}_{2}, 7.5 \mathrm{mg} \mathrm{mL}^{-1}$ lysing enzyme (from Trichoderma harzianum; Sigma L1412), and $3 \mathrm{mg}$ $\mathrm{mL}^{-1}$ cellulase (from Trichoderma reesei; Sigma C8546), and incubated for $40 \mathrm{~min}$ at $25^{\circ} \mathrm{C}$ with $100 \mathrm{r} \mathrm{min}^{-1}$ shaking. The protoplasts were harvested by centrifugation at 1500 $\mathrm{r} \mathrm{min}^{-1}$ for $3 \mathrm{~min}$ and resuspended in W5 solution $(5 \mathrm{mmol}$ $\mathrm{L}^{-1} \mathrm{KCl}, 125 \mathrm{mmol} \mathrm{L}^{-1} \mathrm{CaCl}_{2}, 154 \mathrm{mmol} \mathrm{L}^{-1} \mathrm{NaCl}$, and 31 $\mathrm{mg} \mathrm{mL} \mathrm{m}^{-1}$ glucose) at a concentration of $1 \times 10^{6}$ protoplasts $\mathrm{mL}^{-1}$. After $30 \mathrm{~min}$, the protoplasts were centrifuged at $1500 \mathrm{r} \mathrm{min}^{-1}$ for $4 \mathrm{~min}$ and resuspended in an equal volume of $\mathrm{MMg}$ solution $\left(0.4 \mathrm{~mol} \mathrm{~L}{ }^{-1}\right.$ mannitol, $15 \mathrm{mmol} \mathrm{L}^{-1}$ $\mathrm{MgCl} 2$, and $4 \mathrm{mmol} \mathrm{L}^{-1} \mathrm{MES}$, $\mathrm{pH}$ 5.7) to allow protoplasts to swell. The protoplasts were then diluted to 5000-10000 in each $1 \mathrm{~mL} \mathrm{MMg}$ solution, about $100 \mu \mathrm{g}$ dsRNA was added and incubated for $10 \mathrm{~min}$ on ice. Three $580 \mathrm{~mL}$ aliquots of freshly made polyethylene glycol solution $(40 \%$ (v/v) polyethylene glycol 4000, $0.3 \mathrm{~mol} \mathrm{~L}^{-1}$ mannitol, and $0.15 \mathrm{~mol} \mathrm{~L}^{-1} \mathrm{CaCl}_{2}$ ) were slowly pipetted into the protoplast suspension and gently mixed. After $20 \mathrm{~min}$ incubation on ice, $10 \mathrm{~mL}$ pea broth containing $0.5 \mathrm{~mol} \mathrm{~L}^{-1}$ mannitol was added, and the protoplasts were incubated overnight to re- generate. The regenerated protoplasts were suspended in liquid pea agar $\left(40^{\circ} \mathrm{C}\right)$ containing $0.5 \mathrm{~mol} \mathrm{~L}^{-1}$ mannitol, and then plated. Visible colonies could be observed after $24 \mathrm{~h}$ incubation at $25^{\circ} \mathrm{C}$. A total of 20 single colony transformants were selected and propagated on V8 agar for further testing.

\section{Results}

\subsection{Identification and characterization of $P s C d c 14$ in $P$. sojae}

Using the previously reported amino acid sequence of $P$. infestans Cdc14 [7] as a query, a tBLASTN search program was used to identify a Cdc14 homology from the $P$. sojae genome database (http://genome.jgi-psf.org/Physo1 1/Physo1_1.home.html; March 2009). The gene (protein ID: 108222) was named $P s C d c 14$, and the predicted ORF was 1281 bp, encoding 426 amino acids. The sequence of $\mathrm{PsCdc14}$ transcript was confirmed by sequencing the RT-PCR product, using cDNA from sporulating mycelial mRNA as a PCR template. PsCdcl4 and piCdc14 shared $81 \%$ and $86 \%$ identity at DNA and amino acids level, respectively. The PsCds14 amino acid sequence was analyzed through a conserved domain search (CD-search) at http://www.ncbi.nlm.nih.gov/Structure/cdd/wrpsb.cgi (May 2009) [35]. The region between amino acids 219 and 322 was predicted to be a dual specificity phosphatase catalytic domain, showing about $70 \%$ similarity with the Cdc14 motif from $S$. cerevisiae and other species. The region between the 275 and 285 amino acid sites was a $100 \%$ match with the tyrosine phosphatase active site (VHCKAGLGRTG) (Figure1). As CDC14 is conserved in eukaryotes, phylogenetic analysis of Ykt6 proteins was performed. This revealed that Phytophthora CDC14 is closer to the CDC14 in plants than it is to that in fungi (Figure 2).

\subsection{Expression analysis of $P s C d c 14$ at different life stages of $P$. sojae}

Real time quantitative reverse transcription polymerase chain reaction (qRT-PCR) was used to characterize expression profiles of $P s C d c 14$. RNA samples were isolated from cultured mycelia, sporulating mycelia, zoospores, cysts, germinating cysts, and susceptible soybean tissues infected with P. sojae strain P6497, at 1.5, 3, 6, 12, 24, and 48 hpi. $\mathrm{PsCdc14}$ was expressed at relatively low levels at mycelium and infection stages; however, its expression dramatically increased up to 180-fold in sporulating mycelia, 250-fold in zoospores, and over 1100-fold in cysts (Figure 3).

\subsection{Transient silencing of $P s C d c 14$ expression}

To ensure the specificity of dsRNA-mediated gene silencing, the synthesized dsRNA should not share more than $20 \mathrm{bp}$ 


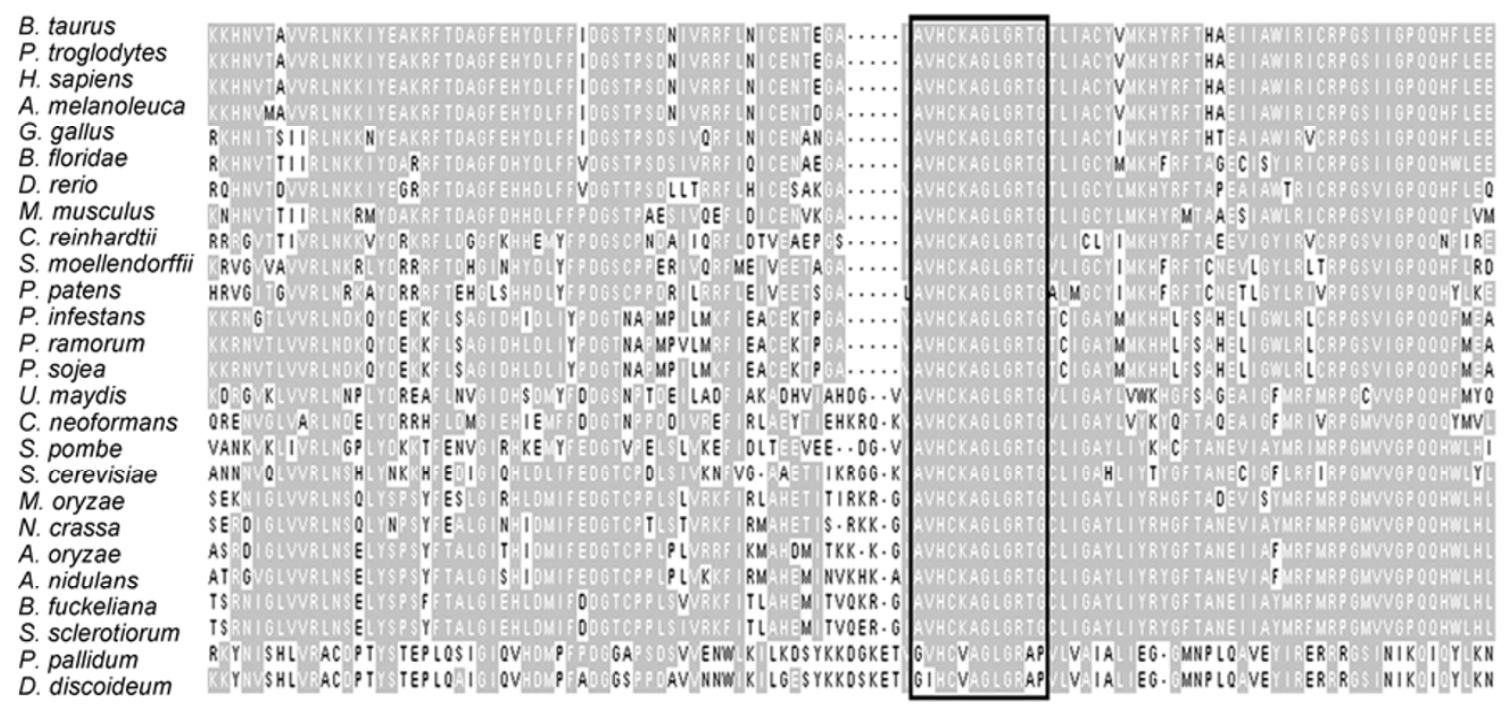

Figure 1 ClustalW alignment of conserved acid amino sequences (segments) of Cdc14 dual specificity phosphatase catalytic domain, from Bos taurus (XP_002686214), Pan troglodytes (XP_001135275), Homo sapiens (ABF74568), Ailuropoda melanoleuca (XP_002920815), Gallus gallus (NP_001171207), Branchiostoma floridae (XP_002602511), Danio rerio (CAP09233), Mus musculus (NP_001116461), Chlamydomonas reinhardtii (XP_001699757), Selaginella moellendorffii (XP_002969311), Physcomitrella patens (XP_001773592), Phytophthora infestans (AAP38170), Phytophthora ramorum (71621), Phytophthora sojae (108222), Ustilago maydis (XP_762334), Cryptococcus neoformans (XP_566871), Schizosaccharomyces pombe (NP_594716), Saccharomyces cerevisiae (YFR028C), Magnaporthe oryzae (XP_362192), Neurospora crassa (XP_963738), Aspergillus oryzae (XP_001819929), Aspergillus nidulans (XP_662661), Botryotinia fuckeliana (XP_001548549), Sclerotinia sclerotiorum (XP_001586094), Polysphondylium yallidum (330787), Dictyostelium discoideum (DDB0238561). The box indicates the tyrosine phosphatase active site.

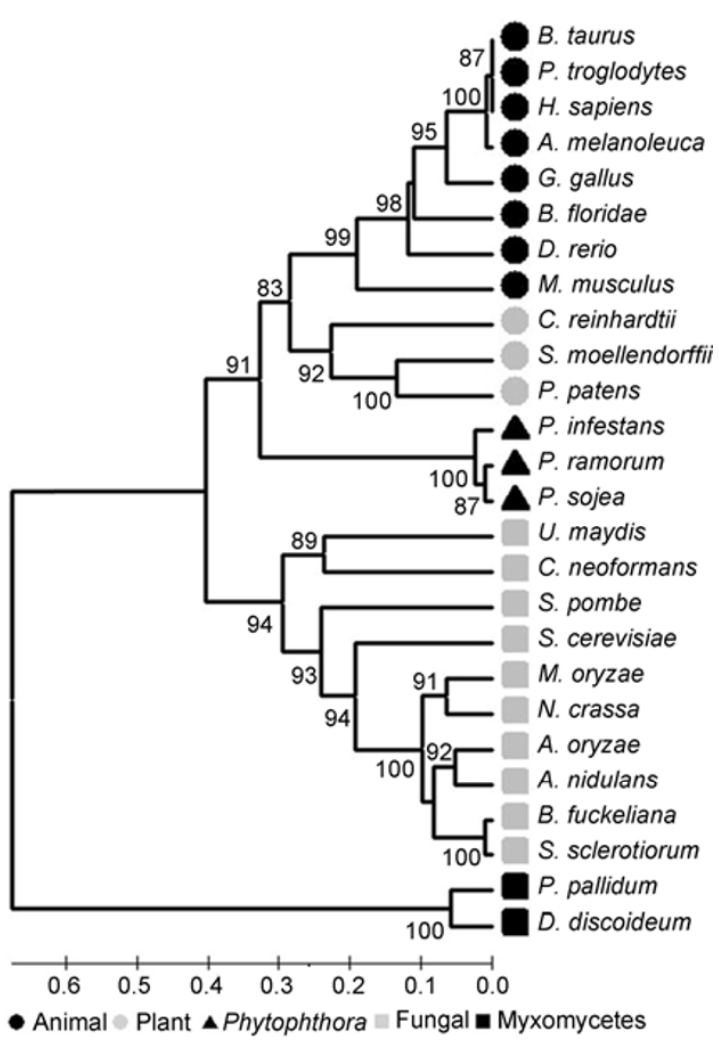

Figure 2 Phylogenetic analysis of Cdc14. Dendrogram of Cdc14 sequences derived from Phytophthora and several other eukaryotes. Alignments were made using ClustalW, and dendrograms produced using Mega4.

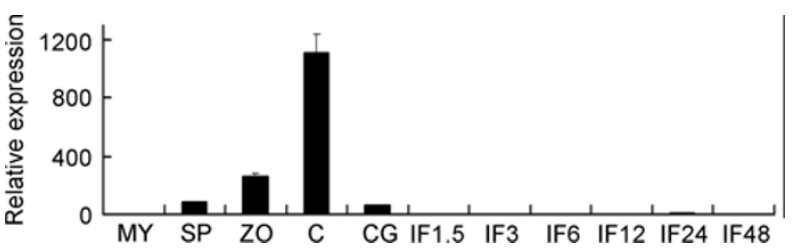

Figure 3 PsCdc14 expression at different developmental and infection stages, as monitored by qRT-PCR. MY, Mycelium; SP, sporulating mycelium; ZO, mixed zoospores and cysts; C, cysts; CG, germinating cysts; IF1.5, IF3, IF6, IF12, IF24 and IF48, infected susceptible soybean cultivar samples at $1.5,3,6,12,24$, and 48 hours post-inoculation, respectively. Expression was equilibrated based on the expression level in mycelium, set at a value of 1 . The qRT-PCR experiment was repeated twice with independent samples.

identity with any other sequences in the genome of $P$. sojae. According to the genome alignment output from the Joint Genome Institute (JGI) P. sojae assembly V 1.0 database, the dsRNA was designed for the specific $\mathrm{N}$ terminal portion of $P s C d c 14$ (Figure 4). Transient transformation of $P$. sojae protoplasts was mediated using polyethylene glycol/calcium chloride (PEG) methods [36,37]. P. sojae isolate P6497 was used as the wild type recipient strain and the transformant from the regenerated protoplast without dsRNA was used as the control. In addition to the high expression of $P s C d c 14$ in sporulating mycelia, zoospores, and cysts, the basal levels of $P s C d c 14$ mRNA in mycelia can be detected using qRT-PCR. Subsequently, qRT-PCR was performed with the extracted RNA from new mycelia to determine whether the 
Table 1 Primers used

\begin{tabular}{cccc}
\hline Primer name & Primer sequence & Application & Application size (bp) \\
\hline Cdc14T7F & GTAATACGACTCACTATAGGGTCTCGAGGTGGCGATCGAGTT & dsRNA synthesis & \\
Cdc14R & GCTTCTTGTTGAGTGTCTCGG & & \\
Cdc14F & TCTCGAGGTGGCGATCGAGTT & dsRNA synthesis & \\
Cdc14T7R & GTAATACGACTCACTATAGGGGCTTCTTGTTGAGTGTCTCGG & & 191 \\
Cdc14qRT-F & CATCCGCAGCATTATGGTCC & Real-time PCR & 134 \\
Cdc14qRT-R & AGCGCATGTACTTCTGGTCG & & \\
ActA qRT-F & ACTGCACCTTCCAGACCATC & Real-time PCR & 165 \\
ActA qRT-F & CCACCACCTTGATCTTCATG & & \\
\hline
\end{tabular}
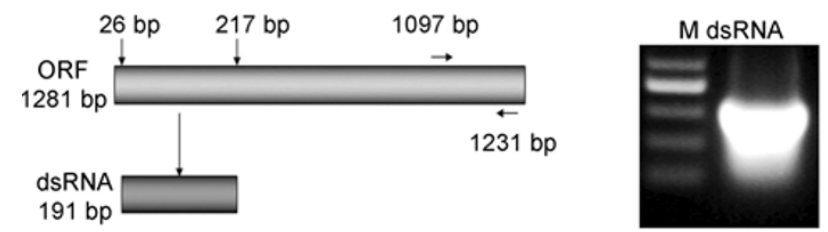

Figure 4 The synthesis of $P s C d c 14$ dsRNA and qRT-PCR primers. A, A $191 \mathrm{bp}$ fragment from 26 to $217 \mathrm{bp}$ was selected as the dsRNA synthesis region, and the primers located at 1097 and 1231 bp were used for mRNA abundance detection using qRT-PCR. B, In vitro synthesized dsRNA products were examined on ethidium bromide-stained gels.

dsRNA-treated lines were silenced for $P s C d c 14$ expression. Relative expression values were assessed 9 days after exposure to $P s C d c 14$ dsRNA or the control. Results from two independent experiments are presented in Figure 5. The $P s C d c 14$-silenced lines showed a reduction in $P s C d c 14$ mRNA abundance, with mRNA levels ranging from $10 \%$ to $30 \%$ of the controls. Successful silencing of $P s C d c 14$ was therefore achieved using RNA silencing.

\subsection{Phenotypic characterization of $\mathrm{PsCdc14}$-silenced lines}

Colony morphology and growth rates of the PsCdc14silenced lines were similar to those of the wild-type recipient strain P6497 and the control transformant. However, at 10 days after exposure to dsRNA, all the $P s C d c 14$-silenced mutants failed to sporulate. In some lines, no sporangia were observed (T2, T3). Some lines produced immature sporangia that were much smaller than the normal sporangia of the wild type and the control transformant (T1). Some lines formed very few normal sporangia (T4) (Figure 6). These results indicate that the $P s C d c 14$ that was partially silenced by dsRNA-mediated transient transformation was defective in sporulation. $\mathrm{PsCdcl4}$ is thus required for $P$. sojae sporulation.

\section{Discussion}

Completion of the cell cycle requires the temporal and spatial coordination of chromosome segregation with mitotic spindle disassembly and cytokinesis. In Saccharomyces cerevisiae, the protein phosphatase $\mathrm{Cdc} 14$ is a key regulator of late mitotic events [38]. In P. infestans, piCdc14 is essential for effective sporulation [7]. $P s C d c 14$, a $C d c 14$ homo$\log$ with a single copy in $P$. sojae, shares a similar transcriptional pattern to piCdc14 in $P$. infestans. This suggests that $P s C d c 14$ and piCdc14 might play the same role in development and sporulation in each species. Although other predicted $P$. sojae proteins have the conserved tyrosine phosphatase active site that is present in the C-terminal region of Cdc14, the 191 bp synthesized dsRNA was designed at the $\mathrm{N}$-terminal region to ensure target specificity. We found that the PsCdcl4-silenced transformants were impaired in sporangium formation. Few, if any, sporangia were produced by the gene-silenced transformants. Sporangia that did develop were reduced in size compared with the control strains. These results are consistent with previously described $P$. infestans $C d c 14$-silencing, indicating that the gene plays a conserved role in Phytophthora sporangial generation, despite differences in sporangial biology be-

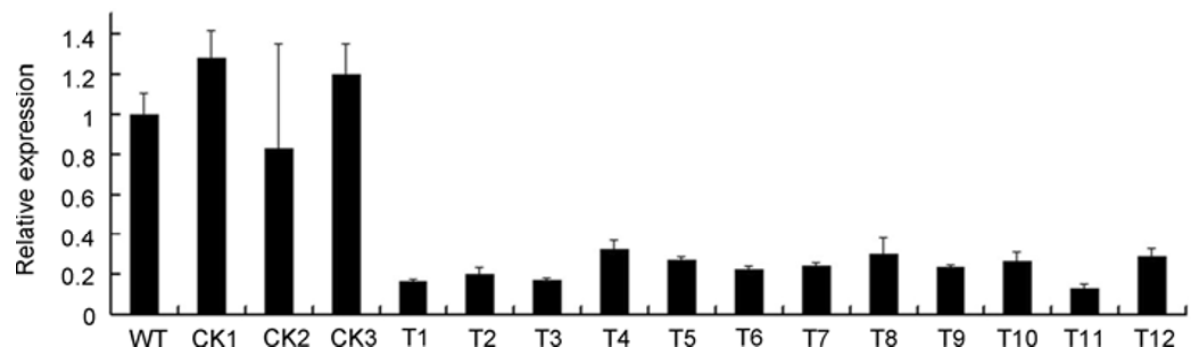

Figure 5 The PsCdc14 relative expression levels in individual RNAi lines were monitored using qRT-PCR. RNA samples were taken from silencing lines 9 days after introduction of PsCdc14 dsRNA into protoplasts. T1-T12 represent the regenerated lines treated by dsRNA. WT is the wild type P6497 strain and CK1-3 is the transformant regenerated without dsRNA introduction. 


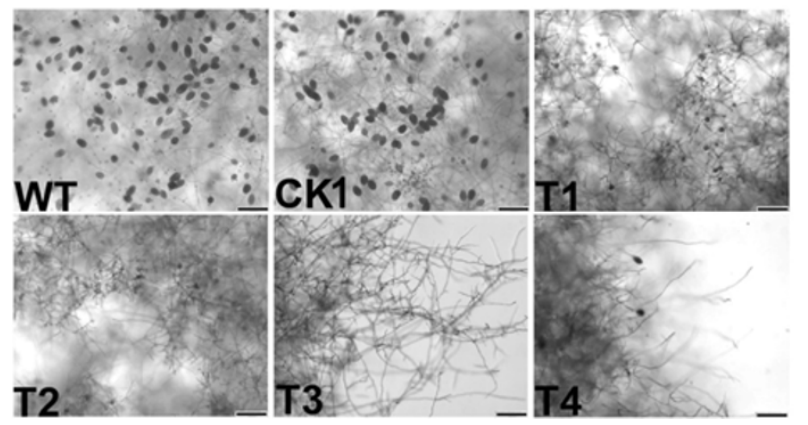

Figure 6 Sporangial formation (bottom) in wild type $P$. sojae (WT), control transformant (CK1), and PsCdc14-silenced mutants (T1-T4). The PsCdc14-silenced lines (T1-T4) showed a significant reduction in the presence or absence of sporangial generation. Scale bars, $100 \mu \mathrm{m}$.

tween the two species.

In other eukaryotes, $\mathrm{Cdc} 14 \mathrm{~s}$ are constitutively transcribed [11]. However, like piCdc14 in P. infestans, PsCdc14 was expressed at a relatively low level in mycelium and infection stages, but its expression dramatically increased in sporulating mycelia, zoospores, and cysts. We propose that the function of $\mathrm{PsCdcl} 14$ may be to synchronize nuclear behavior during sporulation and maintain dormancy in spores until germination. The low level of expression of $P s C d c 14$ in vegetative hyphae might reflect the evolution in some eukaryotes of networks for regulating nuclei that are absent in $P$. sojae. Phytophthora may have lost the normal pattern of Cdc14 expression during its evolution and have adapted to life without a well-regulated cell cycle.

Our dsRNA-mediated gene silencing experiments supported the essential role of $P s C d c 14$ in $P$. sojae sporulation. Assigning a precise role to $P s C d c 14$ is complicated by studies of other species that indicate the protein may have several functions. The specific substrates of Cdc14 that are critical for the decline in Cdk1 activity that accompanies mitosis have been well established. Similarly to Cdk1, Cdc14 has a preference for phospho-Ser/Thr-Pro motifs [39], suggesting that Cdc14 may directly reverse some Cdk1-dependent phosphorylation events. Cdc14 has been shown to dephosphorylate multiple substrates, including Sli15, Ask1, Fin1 and Ase1, which are important for stabilization and extension of the anaphase spindle [40-43]. Cdc14 is also important for the segregation of repetitive ribosomal DNA and telomeric regions of chromosomes during anaphase [44-46], although the relevant target(s) for these processes have not been fully elucidated. In addition, Cdc14 has a role in nuclear positioning and proper segregation of replicated DNA in both the daughter and mother cells [47].

A variety of transformation protocols delivering sense, antisense, and hairpin DNA into Phytophthora cells can result in the silencing of endogenous gene targets in $P$. infestans, P. sojae, and other Phytophthora species [48]. However, the stable transformation of Phytophthora is not only difficult and time-consuming but also may suffer many problems such as a low ratio of gene integration, unstable transformants, lengthy transformation regeneration times, unpredictable copy number, and chromosome insertion effects [48]. Nonetheless, researchers continue to optimize transformation protocols for Phytophthora species, especially $P$. infestans, to simplify and improve the efficiency of transformation [32,34]. A stable transformation method for P. sojae is available and has been employed successfully in the functional characterization of G-protein subunit component PsGPAl, avirulent gene Avrla, transcription factor $P S C R Z 1$, and SNARE protein PsYKT6 [36,37,49,50]. However, the different transformation protocols do not work equally well on different strains of this organism, and adapting improvements described for $P$. infestans or other species are not always successful for $P$. sojae. Current $P$. sojae transformation protocols are thus useful, but need to be improved and expanded to meet the needs of researchers. New and potentially high-throughput tools such as dsRNA-mediated RNAi need to be developed for $P$. sojae. Gene silencing by in vitro synthesized dsRNA (150-300 bp in size), directly delivered into protoplasts with Lipofectin, was first reported in $P$. infestans [32]. A marker gene, GFP, and two $P$. infestans genes, infl and $c d c 14$, were transiently silenced. A reduction of target gene mRNA and a detectable phenotype was observed 12-17 days after introduction of the appropriate dsRNA. Based on previous reports and our own experience, we combined the dsRNA method with a PEG mediated $P$. sojae transformation method, generating a convenient and cost-effective RNAi transient silencing protocol. Whether PEG or Lipofectin is more efficient in mediating dsRNA delivery for Phytophthora is not known since we did not directly compare the two compounds. Nonetheless, from our work on $P s C d c 14$, we have demonstrated that PEG-mediated protoplast exposure to dsRNA triggers gene silencing with resulting phenotypes. This transient gene- silencing protocol is a simple but powerful tool that will assist functional genomics in $P$. sojae. An advantage of the transient silencing of $P$. sojae compared with stable transformation is that there is no need to construct silencing vectors. The simple requirement is the production of dsRNA from a PCR product with T7 RNA polymerase binding sites at each end. Thus, our results substantiated and expanded upon claims that dsRNA-directed RNAi offers a rapid and convenient method to silence target genes in oomycete species.

In summary, we have created the first transient silencing protocol for $P$. sojae, and successfully silenced PsCdc14. We have shown that silencing $P s C d c 14$ results in phenotypic changes in transformants, which affect sporulation, and follow the predicted function of this gene. Our described methods are important for Phytophthora researchers and will enable more feasible studies of gene function. 
This work was supported by the Special Fund for Agro-Scientific Research in the Public Interest (Grant No. 3-20) from the Chinese government, and a project funded by the Priority Academic Program Development for Jiangsu Higher Education Institutions.

1 Tyler B M. Molecular basis of recognition between Phytophthora pathogens and their hosts. Annu Rev Phytopathol, 2002, 40: 137-167

2 Erwin D C, Ribiero O K. Phytophthora Diseases Worldwide. St. Paul: APS Press, 1996

3 Tyler B M. Phytophthora sojae: root rot pathogen of soybean and model oomycete. Mol Plant Pathol, 2007, 8: 1-8

4 Dorrance A E, Mills D, Robertson A E, et al. Phytophthora root and stem rot of soybean. Plant Health Instruct, 2007, doi: 10.1094/PHI-I2007-0830-07

5 Schmitthenner A F. Problems and progress in control of Phytophthora root rot of soybean. Plant Dis, 1985, 69: 362-368

6 Judelson H S, Blanco F A. The spores of Phytophthora: weapons of the plant destroyer. Nat Rev Microbiol, 2005, 3: 47-58

7 Ah Fong A M, Judelson H S. Cell cycle regulator Cdc14 is expressed during sporulation but not hyphal growth in the fungus-like oomycete Phytophthora infestans. Mol Microbiol, 2003, 50: 487-494

8 Latijnhouwers M, Govers F. A Phytophthora infestans G-protein $\beta$ subunit is involved in sporangium formation. Eukaryot Cell, 2003, 2: 971-977

9 Schild D, Byers B. Diploid spore formation and other meiotic effects of two cell-division-cycle mutations of Saccharomyces cerevisiae. Genetics, 1980, 96: 859-876

10 Taylor G S, Liu Y, Baskerville C, et al. The activity of Cdc14p, an oligomeric dual specificity protein phosphatase from Saccharomyces cerevisiae, is required for cell cycle progression. J Biol Chem, 1997, 272: 24054-24063

11 Stegmeier F, Amon A. Closing mitosis: the functions of the Cdc14 phosphatase and its regulation. Annu Rev Genet, 2004, 38: 203-232

12 Visintin R, Craig K, Hwang E S, et al. The phosphatase Cdc14 triggers mitotic exit by reversal of Cdk-dependent phosphorylation. Mol Cell, 1998, 2: 709-718

13 Khmelinskii A, Schiebel E. Assembling the spindle midzone in the right place at the right time. Cell Cycle, 2008, 7: 283-286

14 Hall M C, Jeong D E, Henderson J T, et al. Cdc28 and Cdc14 control stability of the anaphase-promoting complex inhibitor Acm1. J Biol Chem, 2008, 283: 10396-10407

15 Marston A L, Lee B H, Amon A. The Cdc14 phosphatase and the FEAR network control meiotic spindle disassembly and chromosome segregation. Dev cell, 2003, 4: 711-726

16 Buonomo S B C, Rabitsch K P, Fuchs J, et al. Division of the nucleolus and its release of CDC14 during anaphase of meiosis I depends on separase, SPO12, and SLK19. Dev cell, 2003, 4: 727-739

17 Jin F, Liu H, Liang F S, et al. Temporal control of the dephosphorylation of $\mathrm{Cdk}$ substrates by mitotic exit pathways in budding yeast. Proc Natl Acad Sci USA, 2008, 105: 16177-16182

18 Bloom J, Cross F R. Novel role for Cdc14 sequestration: Cdc14 dephosphorylates factors that promote DNA replication. Mol Cell Biol, 2007, 27: 842-853

19 Queralt E, Uhlmann F. Cdk-counteracting phosphatases unlock mitotic exit. Cur Opin Cell Biol, 2008, 20: 661-668

20 Wang Y, Shirogane T, Liu D, et al. Exit from exit: resetting the cell cycle through Amn1 inhibition of G protein signaling. Cell, 2003, 112: 697-709

21 Visintin C, Tomson B N, Rahal R, et al. APC/C-Cdh1-mediated degradation of the Polo kinase Cdc5 promotes the return of $\mathrm{Cdc} 14$ into the nucleolus. Genes Dev, 2008, 22: 79-90

22 McDonald C M, Cooper K F, Winter E. The ama1-directed anaphase-promoting complex regulates the Smk1 mitogen-activated protein kinase during meiosis in yeast. Genetics, 2005, 171: 901-911

23 Holt L J, Hutti J E, Cantley L C, et al. Evolution of Ime2 phosphorylation sites on Cdk1 substrates provides a mechanism to limit the effects of the phosphatase Cdc14 in meiosis. Mol cell, 2007, 25: 689-702
24 Patterson K I, Brummer T, O'Brien P M, et al. Dual-specificity phosphatases: critical regulators with diverse cellular targets. Biochem J, 2009, 418: 475-489

25 Trautmann S, McCollum D. Distinct nuclear and cytoplasmic functions of the $S$. pombe Cdc14-like phosphatase Clp1p/Flp1p and a role for nuclear shuttling in its regulation. Curr biol, 2005, 15: 1384-1389

26 Gruneberg U, Glotzer M, Gartner A, et al. The CeCDC-14 phosphatase is required for cytokinesis in the Caenorhabditis elegans embryo. J Cell Biol, 2002, 158: 901-914

27 Mello C C, Conte D. Revealing the world of RNA interference. Nature, 2004, 431: 338-342

28 Fire A, Xu S, Montgomery M K, et al. Potent and specific genetic interference by double-stranded RNA in Caenorhabditis elegans. Nature, 1998, 391: 806-811

29 Avrova A O, Whisson S C, Pritchard L, et al. A novel non-protein-coding infection-specific gene family is clustered throughout the genome of Phytophthora infestans. Microbiology, 2007, 153: 747-759

30 Grenville-Briggs L J, Anderson V L, Fugelstad J, et al. Cellulose synthesis in Phytophthora infestans is required for normal appressorium formation and successful infection of potato. Plant Cell, 2008, 20: 720-738

31 Walker C A, Köppe M, Grenville-Briggs L J, et al. A putative DEAD-box RNA-helicase is required for normal zoospore development in the late blight pathogen Phytophthora infestans. Fungal Genet Biol, 2008, 45: 954-962

32 Whisson S C, Avrova A O, Van West P, et al. A method for double-stranded RNA-mediated transient gene silencing in Phytophthora infestans. Mol Plant Pathol, 2005, 6: 153-163

33 Chen X, Shen G, Wang Y L, et al. Identification of Phytophthora sojae genes upregulated during the early stage of soybean infection. FEMS Microbiol Lett, 2007, 269: 280-288

34 McLeod A, Fry B A, Zuluaga A P, et al. Toward improvements of oomycete transformation protocols. J Eukaryot Microbiol, 2008, 55: 103-109

35 Marchler-Bauer A, Bryant S H. CD-Search: protein domain annotations on the fly. Nucleic Acids Res, 2004, 32: 327-W331

36 Zhao W, Dong S M, Ye W W, et al. Genome-wide identification of Phytophthora sojae SNARE genes and functional characterization of the conserved SNARE PsYKT6. Fungal Genet Biol, 2011, 48: 241-251

37 Hua C, Wang Y, Zheng X, et al. A Phytophthora sojae G-protein $\alpha$ subunit is involved in chemotaxis to soybean isoflavones. Eukaryot Cell, 2008, 7: 2133-2140

38 Trautmann S, McCollum D. Cell cycle: new functions for Cdc14 family phosphatases. Curr Biol, 2002, 12: R733-R735

39 Gray C H, Good V M, Tonks N K, et al. The structure of the cell cycle protein $\mathrm{Cdc} 14$ reveals a proline-directed protein phosphatase. EMBO J, 2003, 22: 3524-3535

40 Pereira G, Schiebel E. Separase regulates INCENP-Aurora B anaphase spindle function through Cdc14. Science, 2003, 302: 2120_ 2124

41 Woodbury E L, Morgan D O. Cdk and APC activities limit the spindle-stabilizing function of Fin1 to anaphase. Nat Cell Biol, 2007, 9: 106-112

42 Higuchi T, Uhlmann F. Stabilization of microtubule dynamics at anaphase onset promotes chromosome segregation. Nature, 2005, 433: $171-176$

43 Khmelinskii A, Lawrence C, Roostalu J, et al. Cdc14-regulated midzone assembly controls anaphase B. J Cell Biol, 2007, 177: 981993

44 D'Amours D, Stegmeier F, Amon A. Cdc14 and condensin control the dissolution of cohesin-independent chromosome linkages at repeated DNA. Cell, 2004, 117: 455-469

45 Sullivan M, Higuchi T, Katis V L, et al. Cdc14 phosphatase induces rDNA condensation and resolves cohesin-independent cohesion during budding yeast anaphase. Cell, 2004, 117: 471-482

46 Machín F, Torres-Rosell J, Jarmuz A, et al. Spindle-independent 
condensation-mediated segregation of yeast ribosomal DNA in late anaphase. J Cell Biol, 2005, 168: 209-219

47 Ross K E, Cohen-Fix O. A role for the FEAR pathway in nuclear positioning during anaphase. Dev Cell, 2004, 6: 729-735

48 Ah-Fong A M, Bormann-Chung C A, Judelson H S. Optimization of transgene-mediated silencing in Phytophthora infestans and its association with small-interfering RNAs. Fungal Genet Biol, 2008,
45: $1197-1205$

49 Dou D, Kale S D, Wang X L, et al. Conserved C-terminal motifs required for avirulence and suppression of cell death by Phytophthora sojae effector Avr1b. Plant Cell, 2008, 20: 1118-1133

50 Wang Y, Dou D L, Wang X L, et al. The PsCZF1 gene encoding a $\mathrm{C} 2 \mathrm{H} 2$ zinc finger protein is required for growth, development and pathogenesis in Phytophthora sojae. Microb Pathog, 2009, 47: 78-86

Open Access This article is distributed under the terms of the Creative Commons Attribution License which permits any use, distribution, and reproduction in any medium, provided the original author(s) and source are credited. 